\title{
Interrogating Business and Public Administration Research Attributes and Variables
}

\author{
Kambidima Wotela \\ WITS Graduate School of Governance, Johannesburg, South Africa \\ Kambidima.wotela@WITS.ac.za \\ Kambidima.wotela.WSG@GMail.com
}

\begin{abstract}
Research proposal presentation or assessment panels and ethics assessment committees almost always ask us why we have included certain questions in our data or information collection instruments as well as why we think such questions will help us realise our research purpose. How can we answer these questions? First, let us step back. Numerous books have described what a conceptual framework is and why we need it. Further, there are several attempts to describe how we can conceptualise conceptual frameworks primarily through reviewing literature. One of the sets of literature that we should review is on attributes or variables, if not both, that are key to the research that we are pursuing. However, we find the discussion on how we should interrogate literature on attributes or variables not exhaustive to adequately guide a research student or a research novice on what they should do. Therefore, this paper proposes how we should explicitly identify, interrogate, and integrate literature on qualitative attributes or quantitative variables that are key to our business and public administration research. Apart from summoning basic systems thinking principles, the paper draws on reverse engineering comments compiled from examiners' reports 1 and consequent discussions with colleagues and students through devising seminars. We believe that the approach we have proposed in this paper will guide business and public administration research students and novices on how they should interrogate literature on attributes or variables that are key to the research that they are pursuing. If they get this right, it would contribute to robustness of their conceptual framework and, therefore, the research report. Before then, it will also empower us to answer the research proposal presentation or assessment panels and ethics assessment committees on why we have included certain questions in our data or information collection instruments and why we think such questions will allow us to achieve our research objectives.
\end{abstract}

Keywords: research conceptualisation, knowledge gap analysis, interpretive and theoretical frameworks, conceptual framework, research attributes and variables, research procedure and methods, research data and information collection instrument, discussing research findings

\section{Background}

More than ever, research proposal presentation or assessment panels and ethics assessment committees are asking 'why we have included certain questions in our data or information collection instruments' as well as 'why we think such questions will help us realise our research purpose'. This paper, therefore, proposes how we should explicitly identify, interrogate, link, and integrate qualitative attributes or quantitative variables that are key to the business and public administration research that we are pursuing. Our suggestions here may apply to any other research (most probably other social sciences and humanities) outside business and public administration. Our approach rests on basic systems thinking principles described in Gharajedaghi (2006) and builds on the work of Wotela $(2016,2017 a)$. It also reverse- engineers comments of examiners' that assess our research student ${ }^{2}$ reports as well as subsequent discussions with colleagues and research students through devising seminars. If successfully implemented, the proposals in this paper should lead to a contextualised, comprehensive, and critical write-up on qualitative attributes or quantitative variables that are key to the research that we are pursuing. In turn, this allows for confidently answering the research proposal presentation or assessment panels and ethics assessment committees.

To begin with, numerous books such as Ravitch and Riggan (2016) and journal articles such as Rocco and Plakhotnik (2009) and Imenda (2014) as well as conference proceedings such as Wotela (2017) have contributed to our understanding of conceptual frameworks. These source have described, debatably so, what conceptual frameworks are and why we need them in an academic research report, it be a thesis or dissertation. Further, they have attempted to describe how we can conceptualise conceptual frameworks. Wotela (2017) points out that literature review is the primary process of conceptualising conceptual frameworks. Conceptual frameworks comprise several subcomponents or sets of literature to review which vary in number from author to author. However, as we discuss in Section 3, we are convinced from the work of Badenhorst (2007) and to some extent

${ }^{1}$ Examiner's reports of some WITS Graduate School of Governance and WITS Graduate School of Business Administration students.

${ }^{2}$ WITS Graduate School of Governance and WITS Business School

ISSN 1477-7029

(C)The Authors

Reference this paper: Wotela, K., 2022. Interrogating Key Business and Public Administration Research Attributes and Variables. The Electronic Journal of Business Research Methods, 20(1), pp. 21-33, available online at www.ejbrm.com 
Ravitch and Riggan (2016) that any conceptual framework should (i.) interrogate literature on the research problem under study before moving onto (ii.) interrogating empirical literature that provides for exposing the theoretical knowledge gap. Thereafter, we should then (iii.) interrogate literature that discusses the attributes (qualitative) and variables (quantitative) that are key to the research under study. The three sets of literature should then provide for interrogating literature on the frameworks, theoretical or otherwise, that we can potentially use to interpret the anticipated empirical research results.

The focus of this paper is the subcomponent of conceptual frameworks that should supposedly focus on interrogating literature on either qualitative attributes or quantitative variables that are key to the research that we are pursuing-again primarily what this discussion is about and why we need it for our research to be comprehensive and critical. Discussing attributes and variables that are key to the research that we are pursuing is important three reasons. First of all, interrogating literature on either qualitative attributes or quantitative variables provides for conceptualising robust conceptual frameworks. Second, such a discussion provides for identifying appropriate frameworks, theoretical or otherwise, that we should use to interpret our empirical research results. Third, the discussion should ideally be the basis for populating the questions in the research data or information collection instruments that we ask our respondents. However, we fail to explicitly identify, interrogate, and integrate attributes and variables that are key to the research that they are pursuing. This shortcoming is exposed when research proposal presentation or assessment panels and ethics assessment committees asks us to substantiate why we have included certain questions in our research data or information collection instrument.

A quick search of literature shows that, other than research supervisor's or promoters notes ${ }^{3}$ whose access is limited, the discussion on identifying, interrogating, linking, and integrating attributes and variables that are key to the research that we are pursuing is lacking. Ravitch and Riggan (2016), Rocco and Plakhotnik (2009), Imenda (2014) as well as Wotela (2016) have, though not as explicitly, discussed attributes and variables and why we need this information when formulating our conceptual framework. Further, in this journal article, Wotela (2016) attempted to discuss how we should interrogate literature on attributes and variables that are key to the research that we are pursuing. However, the discussion omits how we should identify and integrate the discussion on attributes and variables. As a result, Wotela's (2016) discussion has proved to be insufficient and inadequate to successively guide research students and novices seeking to explicitly identify, interrogate, link, and integrate attributes and variables that are key to the research they are pursuing.

The paper begins with describing the qualitative research attributes and quantitative research variables in Section 2. We note that variables are but attributes that we can measure quantitatively. Most discussions of attributes and variables are in the context of research data or information collection instruments. There is little emphasis for the need to interrogate and understand attributes and variables as part of our conceptual framework. This is the problem that this paper speaks to. Section 3 sets the foundation through reviewing the six main components of a research report as well as the four processes in research. Like in our earlier work, we repeat this part not only because it is fundamental to our contribution but because we have continuously changed the way we think about the components and processes in research as we interact with students in various platforms on this subject. Further, we point out the seven subcomponents of the second component of research, that is, the conceptual framework where we also locate the subcomponent (fifth) that interrogates literature on attributes and variables that are key to the research that we are pursuing. Section 4 points out the 'golden threads' in researching and research reporting to emphasise the need to explicitly link the various research components and subcomponents as an avenue of attaining criticality. We then situate this paper in one of the four 'golden threads' as our point of departure.

In Section 5, we discuss with illustrations and examples how to pivot a business and public administration research through identifying, interrogating, and integrating qualitative attributes and quantitative variables that are key to the research that we are pursuing. First, we point out that we should use the research questions as well as where applicable the accompanying research hypotheses or research propositions to identify the attributes or variables, if not both, that are key to the research that we are pursuing. Second, during our 'theoretical' knowledge gap analysis that we undertake as part of literature review to determine additional attributes or variables, if not both, that are key to the research that we are pursuing. Third, we should then

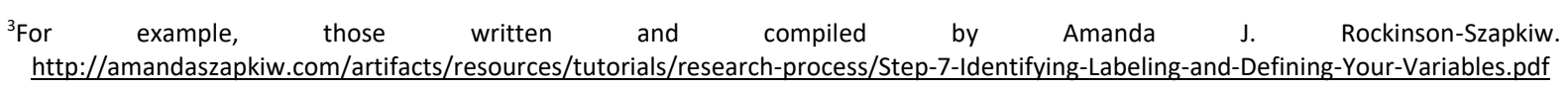


discuss the identified attributes and variables within the broader context of the academic field-of-study that is appropriate to the research that we are pursuing. Fourth, we should use the interrogation of our selected attributes and variables to derive the questions that we should include in our empirical data or information collection instruments that we then use to interview our research responds. Lastly, this discussion should also help us identify the appropriate frameworks, theoretical or otherwise, that we should use to interpret our empirical research results.

\section{Describing research attributes and variables: The missing emphasis}

Almost all research methodology textbooks-such as Neuman (2013), Bryman (2016), and Kumar (2018)-have defined, described, and discussed qualitative research attributes and quantitative research variables. Collectively, attributes and variables-as well as indicators and determinants-are changing or varying characteristics of a given phenomenon. First, this property allows for logically varying categories or characteristics or classifications of a given phenomenon. For example, sex has two categories or characteristics or classifications (girl or woman or female and boy or man or male) whilst classification of residence has three (rural, semi-urban, urban). Therefore, as Kaur (2013) points out, attributes and variables change from one person, organisation, institution, sub-region, region, country, and continent to the next or the other. This implies that not everyone in our research sample would have the same age, sex, height, weight, occupation, income, temperature as well as culture, language, religion, happiness, and anxiety. Second, the dynamic property of attributes and variables allows us to compare the relative positions (good or bad) of different persons, organisations, institutions, sub-regions, regions, countries, or continents on a given phenomenon that we are studying.

The distinction between attributes and variables is that the latter has numerical categories or characteristics or classifications which we can measure quantitatively. However, we can only manipulate the former qualitatively. This means that qualitative attributes become quantitative variables if we can quantitatively measure the change between their respective categories or characteristics or classifications. For example, attributes of public transport systems could be affordability, convenience, flow, and safety. The variables of such attributes could be transport costs, rate of private transport usage, travel timing, travel distance, travel speed, travel duration, and proportion of road accidents.

There are various types of attributes and more so variables such as independent versus dependent as well as extraneous. Kaur (2013) has also differentiated them on their scales of measurement-that is, nominal (attributes), ordinal (attributes with a specified numerical order), interval (numerical), and ratio (numerical). On an operational level, attributes and variables are what we ask our respondents or look for when collecting quantitative data (unorganised information) or qualitative information (organised data) so that we can answer our theoretical research questions or test our theoretical hypotheses or prove our theoretical propositions. This explains why most discussions of attributes and variables is almost always in the context of research data or information collection instruments.

There is no doubt that the foregoing description of attributes and variables is common and prominent in most research methodology textbooks -including but not limited to Neuman (2013), Bryman (2016), and Kumar (2018). However, as stated earlier, this description is in the context of research data or information collection instruments. Therefore, the focus is operationalisation of variables and sometimes, though not as explicit, attributes. There is little emphasis on the need to interrogate and understand attributes and variables-as part of our conceptual framework-before we include them in our research data or information collection instruments. The closest we get is Neuman (2013) who has described with examples how to source, summarise, and synthesis literature. However, even he does not explicitly discuss how we should do this for literature on attributes or variables that are key to the research that we are pursuing. The absence of this articulation is partly why we fail to answer the questions raised by the research proposal presentation or assessment panels and ethics assessment committees as to why we have included certain questions in our research data or information collection instruments as well as why we think such questions will help us realise our research purpose.

It is one thing to know what qualitative research attributes and quantitative research variables are and how they help us with formulating our data or information collection instruments. However, it is another thing to figure out the most appropriate attributes or variables that we should include in the instruments. Therefore, the gist of this paper is to identify, interrogate, and integrate the theoretical foundation underpinning the attributes and 
variables that are key to the research that we are pursuing. This is certainly a useful discussion because whilst some attributes and variables as well as their categorisation or characterisation or classification are straightforward and established, the others are abstract and vague (Kaur, 2013). It is also most likely to help us to answer the questions, 'why we have included certain questions in our data or information collection instruments?' and 'what makes us think that such questions will help us to answer our research questions?'

\section{Research components, subcomponents, and processes}

As we have argued earlier (Wotela, 2016), researching and research reporting is a complex undertaking. Therefore, to resolve this complexity, it helps to approach research from a systems thinking perspective. Identifying components (stock) and processes (flow) of complex phenomena, in this case research, is fundamental to systems thinking (Gharajedaghi's 2006). Using systems thinking approach detailed in Wotela (2016), we have identified and detailed six components of a business and public administration research report (Wotela, 2016, 2019) regardless of the number of chapters as presented in Figure 1, that is:

1. Introduction to the research to conceptualise the research

2. Reviewing literature to derive the conceptual framework

3. Research strategy, design, procedure and methods

4. Presentation of empirical research results

5. Discussion of research findings

6. Summary, conclusions, limitations, and recommendations

Relatedly and closely matching the six components, there are four processes in researching, that is, (i.) research conceptualisation, (ii.) theoretical interrogation, (iii.) empirical interrogation, and (iv.) empirical implementation as well as documentation. Research conceptualisation in business and public administration entails getting a research idea onto its researching trajectory. This involves intense reflection as well as iterative interrogation of the first component of a research report as well as the first three subcomponents of the second component (Wotela, 2019). The product of this process is spelling out explicitly and critically what the research is about and why we should bother to pursue it. The key products of this process are (i.) the research problem statement, (ii.) the research purpose (aim and objectives) statement, (ii.) the research questions as well as where applicable the accompanying research hypotheses or research propositions. The by-products of a thorough research conceptualisation process, which are also key to the entire research undertaking, are (i.) the research problem analysis within its physical context or space or setting and (ii.) the research theoretical knowledge gap analysis or empirical literature review.

Second, theoretical interrogation-which is primarily a structured and critical literature review resulting into a conceptual framework (second component) - provides for determining how we should undertake our research having already determined what research we should pursue and why during research conceptualisation. Among others, the key primary products of a theoretical interrogation process includes (i.) identification and interrogating the attributes or variables, if not both, that are key to the research that we are pursuing and (ii.) identification and interrogating the framework, theoretical or otherwise, that we should use to interpret our empirical research results (Wotela 2016). Eventually, the products arising from the research conceptualisation process and the theoretical interrogation process feed into conceptualising the conceptual framework for the research that we are pursuing.

Third, empirical interrogation is primarily reviewing research methods literature to detail our envisaged research strategy, design, procedure and methods (third component) proposed in our conceptual framework (second component). Lastly, empirical implementation and its documentation is the actual (and documentation of) collecting, collating, processing, and analysing empirical research data or information. We describe this process in the third component alongside the empirical interrogation. Thereafter, we present the empirical research results in the fourth component and-with the aid of the interpretive framework detailed in the second component-we discuss the research findings in the fifth component. In sum, we document the empirical implementation process in the third component and, thereafter, deposit the outputs (empirical research results) in the fourth component and the outcomes in the fifth component (discussion of research findings). 


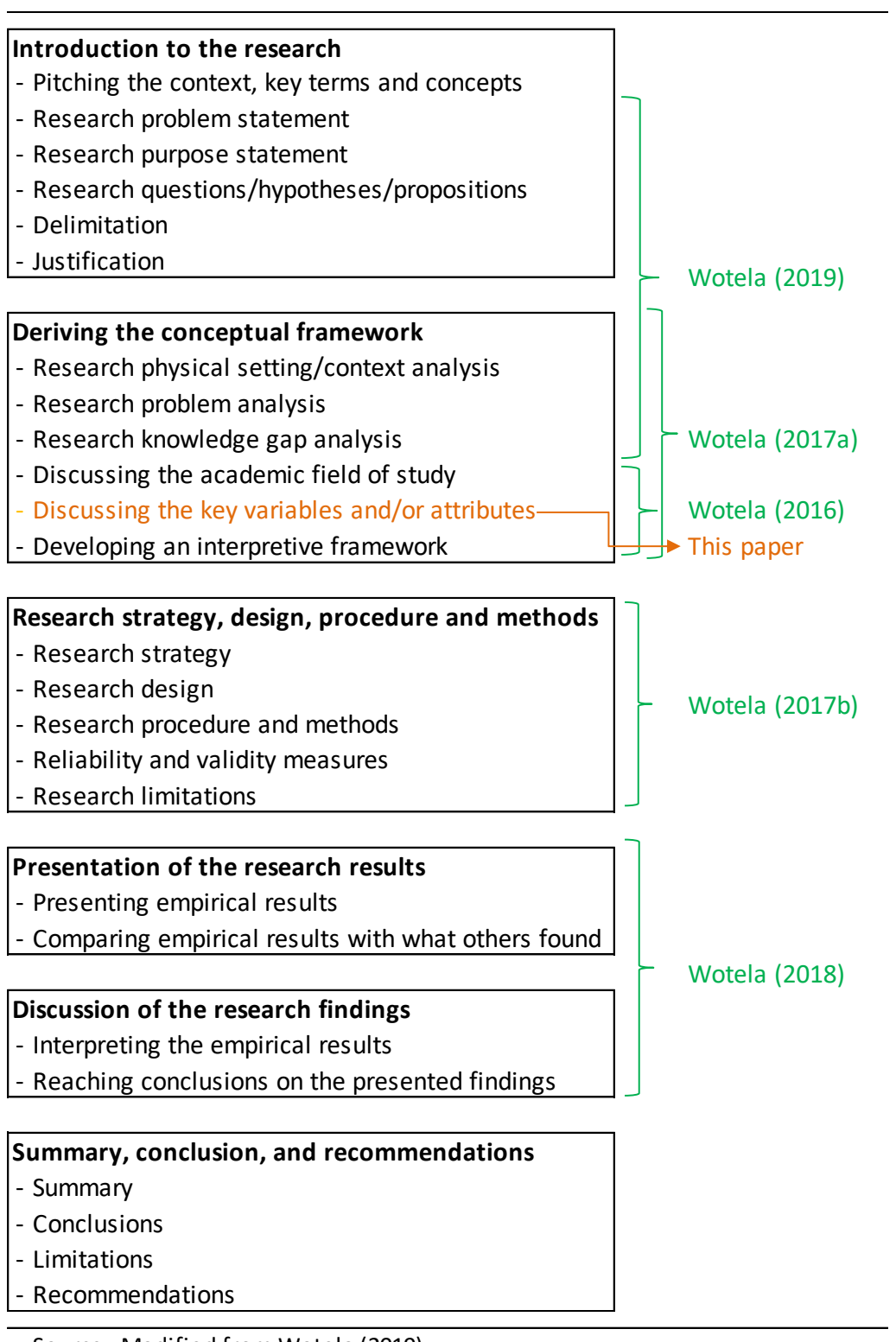

Source: Modified from Wotela (2019)

Figure 1: Showing the six components of a research report and their subcomponents

Apart from the fundamental six components and four processes of a business and public administration research report, Wotela (2016, 2017a, 2017b, 2018, and 2019) has also identified and detailed the first level subcomponents of the six components of research undertaking as presented Figure 1. For this paper, we highlight the subcomponents of the second component-that is, reviewing literature to derive the conceptual framework. He has argued that to derive a conceptual framework for the research that we are pursuing, we have to interrogate following sets of literature;

- 1. Research physical setting or context analysis

- 2. Research problem analysis

- 3. Research knowledge gap analysis

- 4. Establishing and discussing the academic context and its key components

- 5. Establishing and discussing the key research variables and/or attributes

- 6. Establishing and discussing the interpretive framework for the research

Eventually, summarising these six sets of literature provides for the seventh and final subcomponent of the conceptual framework (Wotela, 2016, 2019). Other than summarising the literature reviewed, the seventh component provides for proposing how we should carry out the empirical part of our research on the basis of the literature that we have interrogated. Therefore, as Wotela (2017a) has argued, a conceptual framework is an outline of how we should undertake the empirical part of our research-supported by, or engraved in, the 
six sets of literature that we have interrogated. We should note that the focus of this paper is the fifth subcomponent of a conceptual framework - that is, establishing and discussing the key research attributes or variables, if not both.

Arnold and Wade (2015) state that as with most systems-in our case, the researching and research reporting system-systems thinking consists of three considerations, that is; a function or purpose, elements and components, and the interconnections between the elements and components. Implying from Arnold and Wade (2015), although not all systems have an obvious goal or objective, like systems thinking, a research should always have a defined aim and accompanying objectives. "The ... function or purpose [of a research is its] most crucial determinant ..." (Arnold and Wade, 2015, p3). Generally, this comprises its 'research conceptualisation' component and process (what research to pursue and why) as well as the accompanying conceptual framework component (how to pursue the research) arising from its 'theoretical interrogation' process. The second consideration is 'elements and components' that we detail in this Section. Lastly, it is the interconnections between the research elements and components that describes how they relate to and feed back into each other. We describe the interconnections of the elements and components of a researching and research reporting system in the next Section.

\section{The four 'golden threads' in researching and research reporting}

As provided for in systems thinking, the ability to link, and explicitly so, the various components and subcomponents of a research report provides for rigour and criticality. There could be more but we can claim at least four notable 'golden threads' in humanities and social science research in general and specifically business and public administration. First, (i.) the research title, (ii.) research problem statement, (iii.) research purpose statement, and (iv.) the research questions-as well as where applicable the accompanying research hypotheses or research propositions-should align. This is a fundamental red flag for most examiners as well as members of the research proposal presentation or assessment panels. More generally, they would like to see a harmonised content in the research title, the research problem statement, the research purpose statement, and the research questions. Specifically, between the research problem statement and the research purpose statement as well as between the research purposes statement and the research questions. Lastly, there as to be harmonisation between the research questions as well as where applicable the accompanying research hypotheses or research propositions.

Second, 'the introduction to the research' component should align with 'the summary, conclusions, limitations, and recommendations' component. The former provides for documenting the research conceptualisationspelling out what the research is about and why we should pursue it. Having decoded the how to undertake the research and then moving on to actually implement the research in the subsequent components, it is important to document what the research has consequently realised in ultimate component-that is, 'the summary, conclusions, limitations, and recommendations' component. It is more critical if we deliberately mirror the primordial and ultimate components with the former showing 'what we promised' and the latter showing that 'we have delivered what we promised'. This should be in context of what we actually did to get the empirical research results and reaching the conclusions as well as what these entail.

Third, the research questions and where applicable the accompanying research hypotheses or research propositions should align with the discussion on qualitative attributes or quantitative variables, if not both, that are key to the research that we are pursuing. In turn, the discussion on qualitative attributes or quantitative variables, if not both, should align with the questions in the research data or information collection instrument. Further, this discussion should also align with the frameworks, theoretical or otherwise, for interpreting empirical research results.

Lastly, the frameworks, theoretical or otherwise, for interpreting the empirical research results should align with the discussion of research findings. It is important to discuss relevant frameworks that are key to the research that we are pursuing. However, we should ensure that we actually use these frameworks when we interpret our empirical research results.

The focus of this paper is the third 'golden thread'. In essence, we are pursuing the question, 'how do we get to use the discussion on qualitative attributes or quantitative variables, if not both, to pivot our research so that it relates our (i.) research questions-as well as where applicable the accompanying research hypotheses or 
research propositions-on one hand and on the other hand (ii.) the data or information collection instruments as well as (iii.) the frameworks, theoretical or otherwise, for interpreting empirical research results?'

\section{Identifying, interrogating, and integrating literature on attributes and variables that are key to the research we are pursuing}

To answer the questions, 'why have we included certain questions in our data or information collection instruments?' and 'what makes us think that such questions will help us to answer our research questions?' we should learn to explicitly identify, interrogate, and integrate literature on the attributes and variables that are key to the research that we are pursuing. This includes appreciating how these attributes and variables are related to each other (Kaur, 2013) and how they relate to our research questions as well as the frameworks, theoretical or otherwise, that we will eventually use to interpret the empirical research results. First off, as Wotela $(2016,2018)$ points out, the discussion on attributes or variables that are key to the research that we are pursuing is integral to the conceptual framework. This subcomponent should bridge the four research processes that we describe in Section 2. This means, as Figure 2 illustrates, we should deliberately pivot the discussion in this subcomponent by simultaneously and explicitly taking on board four related tasks of considerations.

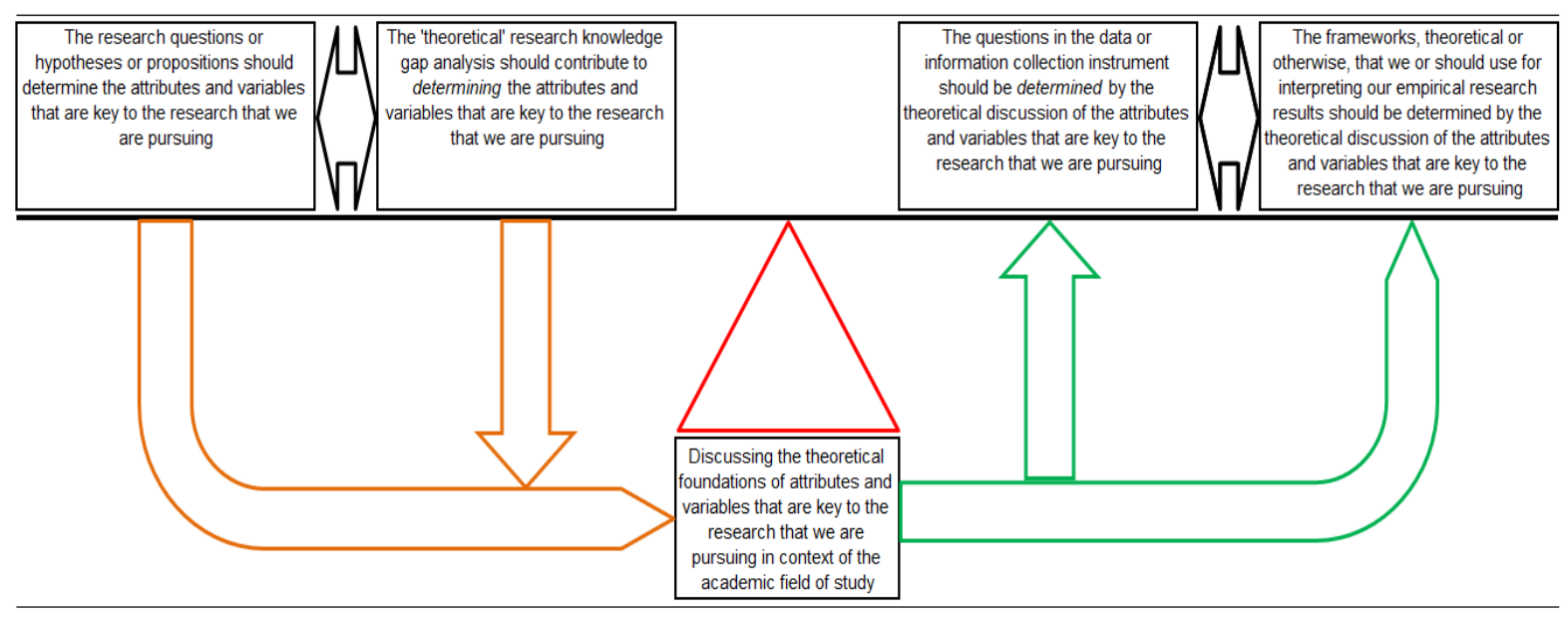

Figure 2: Showing how we should pivot the discussion of attributes and variables that are key to the research we are pursuing

First, (upper far left of Figure 2) the research questions-and where applicable the accompanying research hypotheses or research propositions-should determine the attributes or variables that are key to the research that we are pursuing ${ }^{4}$ (Connelly, 2015; Li \& Ma, 2013). We should therefore begin with reviewing, reflecting, reconceptualising, and editing the research questions that we have posed and where applicable the accompanying research hypotheses or research proposition (Farrugia, et. al., 2010). For example, Table 1 shows the research question (first row) and the accompanying null hypothesis (second row) and alternative hypothesis (third row) as well as the research proposition (fourth row). Interrogating this research question and its accompaniments suggests that 'savings', 'traditional banking institutions', and 'income' as well as qualifying what we mean by 'people' should be the attributes and variables that are key to this research. We should then subject these attributes and variables to literature interrogation so that we have a firm theoretical grounding on them and related factors. Doing so will also provide for identifying the other not so obvious aspects that can help us answer our research questions or test our research hypotheses or prove our research propositions. Inherently, such an undertaking provides for explicitly linking the research questions and where applicable their accompaniments (presented in the first component of the research report) ${ }^{5}$ to the discussion of attributes and variables that are key to the research that we are pursuing (presented in the second component of the research report $)^{6}$.

\footnotetext{
${ }^{4}$ In some cases, this could also be vice versa.

${ }^{5}$ As we presented in Section 2, the first component of a research report-the introduction to the research-is dedicated to conceptualising 'what' research we are undertaking and 'why' we should bother to do so.

${ }^{6}$ The second component of a research report-reviewing literature to derive a conceptual framework-is dedicated to interrogate literature so that we figure out 'how' we should undertake the research that we are pursuing.
} 
Table 1: Showing the research questions and the accompanying research hypotheses and research propositions as well as the arising attributes and variables

\begin{tabular}{|c|c|}
\hline $\begin{array}{l}\text { Research questions and accompanying research } \\
\text { hypotheses or research proposition }\end{array}$ & $\begin{array}{l}\text { Identified research attributes and/or } \\
\text { variables }\end{array}$ \\
\hline $\begin{array}{l}\text { What factors hinder people from saving in traditional } \\
\text { banking institutions? }\end{array}$ & \multirow{4}{*}{$\begin{array}{l}\text { \#People } \\
\text { \#Saving } \\
\text { \#Traditional banking institutions } \\
\text { \#Income }\end{array}$} \\
\hline $\begin{array}{l}\text { There is no difference between higher income } \\
\text { earners and lower income earners in the proportion } \\
\text { of money they save. }\end{array}$ & \\
\hline $\begin{array}{l}\text { The proportion of money saved by higher income } \\
\text { earners is different from the proportion of money } \\
\text { saved by lower income earners. }\end{array}$ & \\
\hline $\begin{array}{l}\text { If people had high incomes, they would save in } \\
\text { traditional banking institutions. }\end{array}$ & \\
\hline
\end{tabular}

Second, (upper middle left of Figure 2) the 'theoretical' research knowledge gap analysis that we undertake as part of literature review should also contribute to determining the attributes or variables that are key to the research that we are pursuing (Zhao, Wu, and Liu, 2016). This is also called empirical literature review and involves reviewing past and current research that is similar to the one we are pursuing. The literature sources for this interrogation are primary or empirical research implying that the researchers or the authors actually collected, collated, processed, and analysed empirical research data or information whose results they consequently presented and discussed the findings. Obviously, they employed attributes or variables to collect, collate, process, and analyse empirical research data or information for their research. We can adopt as well as adapt some of their attributes and variables if they are key to the research that we are pursuing. For example, in addition to the four attributes and variables that we have identified in Table 1, reviewing past and current research can point to including consumption. These studies would argue that it is difficult to talk about savings without referring to income and consumption. Again, we should then subject the adopted and adapted attributes and variables to literature interrogation so that we have a firm theoretical grounding on them and other related factors. This undertaking provides for linking our (i.) research questions and where applicable their accompaniments (presented in the first component of the research report) to (ii.) the research knowledge gap analysis or empirical literature review as well as (iii.) the discussion of attributes and variables that are key to the research that we are pursuing (presented in the second component of the research report).

Third, (bottle middle part of Figure 2) after determining the attributes or variables that are key to the research that we are pursuing through (i.) the research questions as well as where applicable their accompaniments and (ii.) the 'theoretical' research knowledge gap analysis or empirical literature review, we should subject them to a literature interrogation. However, a theoretical discussion of the selected attributes and variables should be in the broader context of the academic field-of-study relevant to the research that we are pursuing. Indeed, to answer research questions or test research hypotheses or prove research propositions, we should collect the views and opinions of our research respondents through our data or information collection instruments. Regardless, the questions that we pose to our respondents in these instruments should have a firm theoretical and probably practical grounding. For example, unknowingly or otherwise, the issues that influence the respondents' views and opinions on the services that learning institutions deliver sits in literature on education in general and specifically the education service delivery attributes and variables. Therefore we should, before anything else, have a theoretical understanding of education as the academic context and, thereafter, the education service delivery attributes and variables. In reference to Table 1, it is clear that the academic context would be economics. Therefore, we should interrogate literature to have a theoretical understanding of economics in general and then microeconomics (or macroeconomics) and, thereafter, the key attributes and variables in economics that are key to our research - that is; household income, consumption, savings as well as traditional banking institutions-all in the context of the 'people' that we are studying. This certainly provides us with a solid theoretical foundation on the variables that are key to our research on savings. We should also include a discussion of the various sources of data and information on these attributes and variables. 
Further, our interrogation of attributes and variables should allow us to theoretically understand them individually and how they interlink or relate to each other as well as how they link in with the key aspects of the research that we are pursuing. Figures $3 \mathrm{~A}$ and $3 \mathrm{~B}$ show that it is helpful to map these relationships to bring out the causal-logical relationships between attributes or variables. Figure $3 A$ shows that we can get to attributes or variables by breaking down the relevant field-of-study into components and further its components into components until we get to elements that we can collect data and information on. Furthermore, unlike Figure $3 \mathrm{~A}$, Figure $3 \mathrm{~B}$ shows that the outcome may not always be pyramid or semi-pyramid shaped. In all cases, we should use literature to determine or support the identified components and subcomponents as well as the resulting attributes and variables. The literature should also determine or support the relationships between attributes and variables. Wotela (2016) has proposed how we should interrogate literature on the academic field-of-study, its components as well as its attributes and variables. Crossan and Apaydin's (2010) paper is another compelling example of what we should aim to achieve in this interrogation.

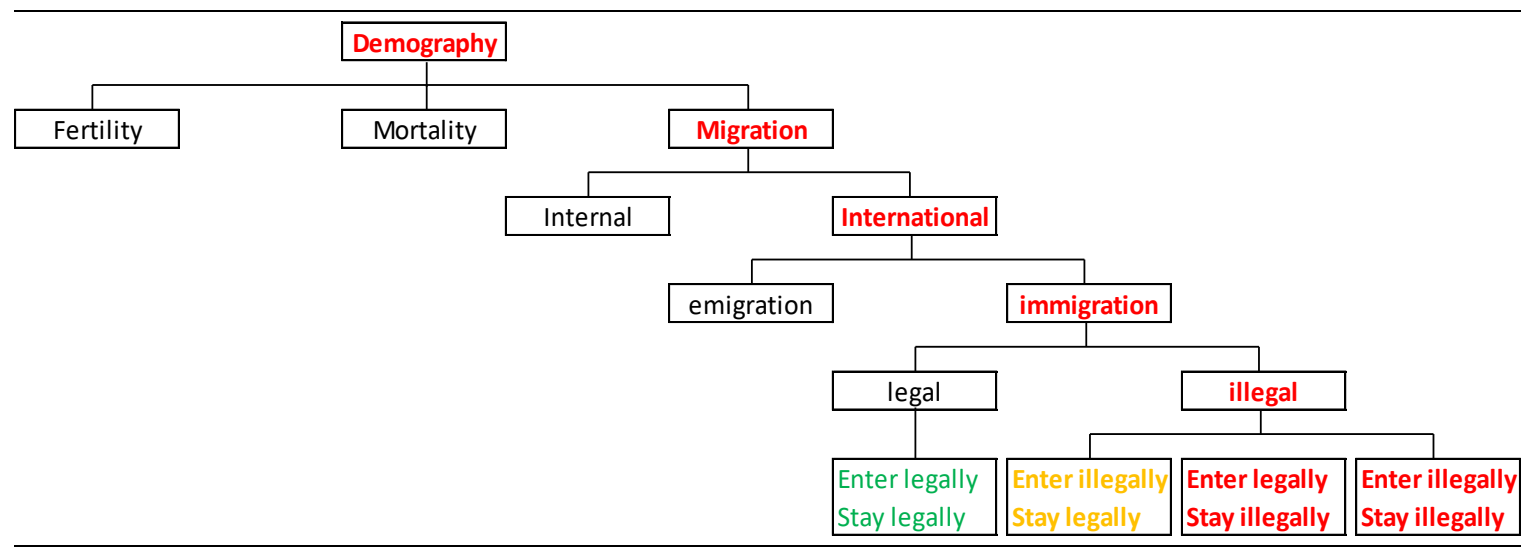

Figure 3A: Showing the academic field-of-study (demography), its key components at various levels, and the attributes or variables for studying illegal international migration (Letsiri \& Wotela, 2015)

Fourth, (upper middle right of Figure 2) we should use the discussion of qualitative attributes and quantitative variables to determine the research strategy, design, procedure and methods (the third component of a research report) that we will employ for our research. More specifically, it should determine the questions that we field in our research data or information collection instrument (Pushpanjali, Piddennavar, and Mohan, 2011). Therefore, there should be a link, explicit for that matter, between the theoretical discussion of the attributes and variables that are key to the research that we are pursuing on one hand and on the other hand the questions that we should pose to our research respondents to elicit empirical research data or information from them. Inherently, this links our research questions (and where applicable the accompanying research hypotheses or research propositions) and the questions that we package in our data or information collection instruments. Further, this link is anchored by our theoretical understanding of the attributes or variables that we have selected.

To illustrate, Figure 4 shows what we have stated earlier that we should interrogate economics in general and then microeconomics (or macroeconomics) and thereafter its variables-household income, consumption, and savings to get a solid theoretical foundation on the variables that are key to our research on savings. We should obviously extend this theoretical interrogation to traditional banking institutions and context, that is, the 'people' that we are studying. This certainly provides us with a solid theoretical foundation on the variables that are key to our research on savings. Further, we should then use our selected and theoretically interrogated attributes and variables to derive the questions that we should ask our research respondents-in this case about their household income as well as their consumption and savings habits. Although the questions that we pose to our respondents are 'ask-able' - that is, questions that non-specialists can actually comprehend and respond to-cumulatively, they help us answer our 'theoretical' research questions. For example, we cannot ask our respondents about their microeconomic status but we can ask them about their income, consumption, and savings. From their answers, we can then establish their micro-economic status using our theoretical and practical understanding of economics, its components, as well as its attributes and variables-more so how these variables are inter related. 


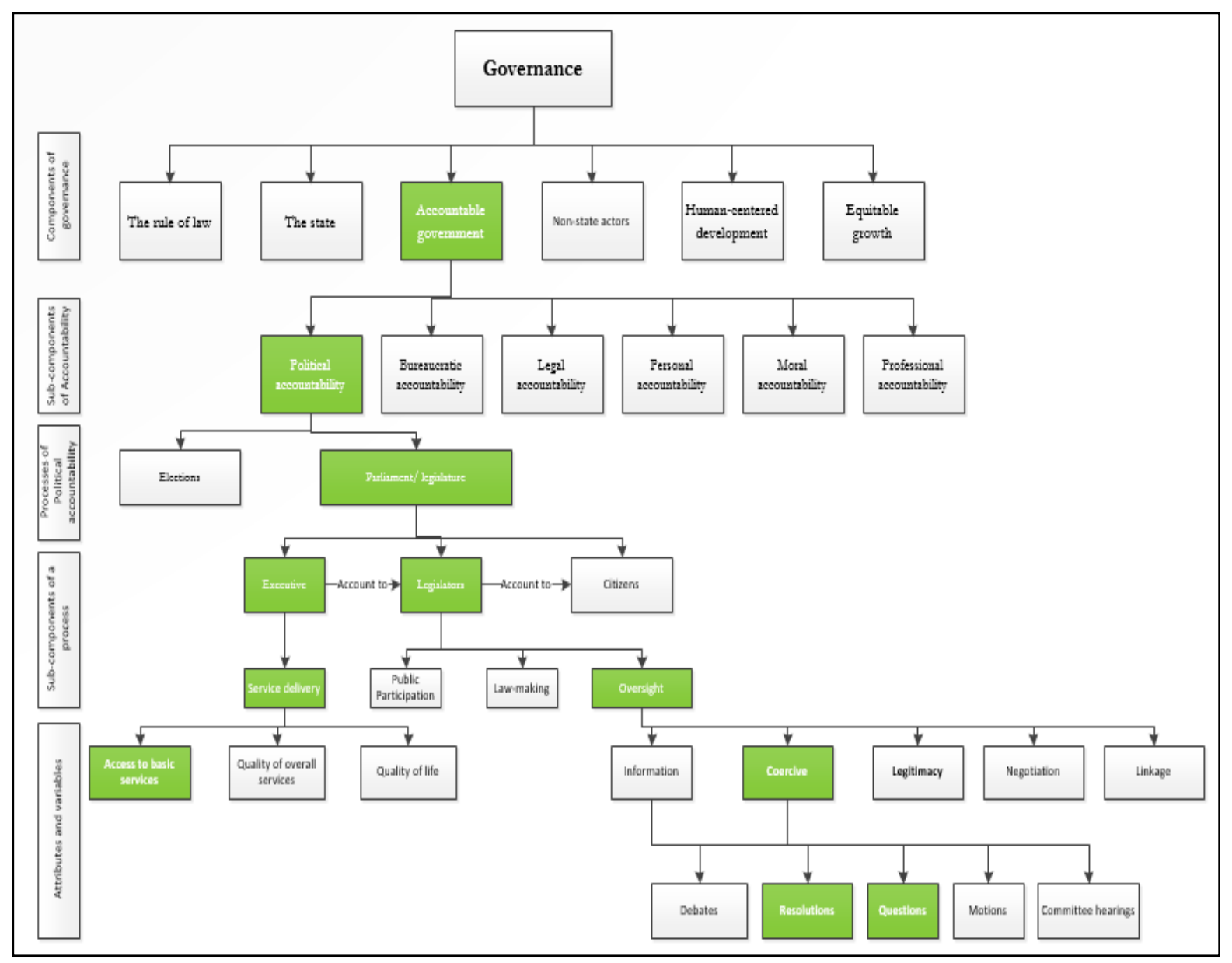

Figure $\mathbf{B B}^{7}$ : Showing the components, attributes, and variables of legislative oversight and service delivery within the governance academic field-of-study

Inherently, EVERY question in our data or information collection instrument should emanate from a thorough and explicit theoretical interrogation within an appropriate academic field-of-study. Therefore, as part of our conceptual framework, we are theoretically interrogating attributes and variables that are key to the research that we are pursuing to pivot our research. On one hand, our selected attributes and variables are linked to (i.) the research questions and where applicable the relevant accompaniments and (ii.) the 'theoretical' knowledge gap analysis or empirical literature review while, on the other hand, they are linked to the questions in our data or information collection instruments that we will use to collect data or information from our research respondents. Such a deliberate link will certainly provide for answering the questions, 'why we have included certain questions in our data or information collection instrument?' and 'why we think such questions will help us realise our research purpose?' Further and more generally, this interrogation may also provide valuable input into how we should collect, collate, process, and analyse empirical research data or information that we will collect using the questions in our research data or information collection instruments (Wotela, 2017b).

Lastly (upper far right of Figure 2) we should use (i.) the discussion of qualitative attributes and quantitative variables and (ii.) the questions that we populate in our empirical data or information collection instrument to determine the most appropriate frameworks, theoretical or otherwise, that we should consider for interpreting our empirical research results (Marzo-Navarro, Pedraja-Iglesias, and Vinzón, 2017). When interrogating literature on the attributes or variables that are key to the research that we are pursuing, we might encounter frameworks that are relevant to our selected attributes or variables, if not both. For example, we are likely to find a discussion on microeconomic theory when we are interrogating literature on income, consumption, and savings. The other way round is that literature on frameworks of interest, would also point to attributes and variables that maybe key to the research that we are pursuing. For example, literature on microeconomic theory

\footnotetext{
${ }^{7}$ Credit: Mokibelo R. Ntshabeleng's research on 'Correlating effective legislative oversight and improved service delivery: A case of Gauteng Province'
} 
might point to assertions - such as, 'all things being equal, higher income leads a higher propensity to spend' and, therefore, inherently pointing out income, consumption, and savings as important attributes or variables. Doing so will allow us to link (i.) the research questions and where applicable the accompanying research hypotheses or research propositions, (ii.) the attributes or variables, if not both, that are key to the research that we are pursuing, (iii.) the questions that we should ask our respondents to elicit empirical research data or information from them, and (iv.) the framework, theoretical or otherwise, that we should consider to interpret our empirical research results.

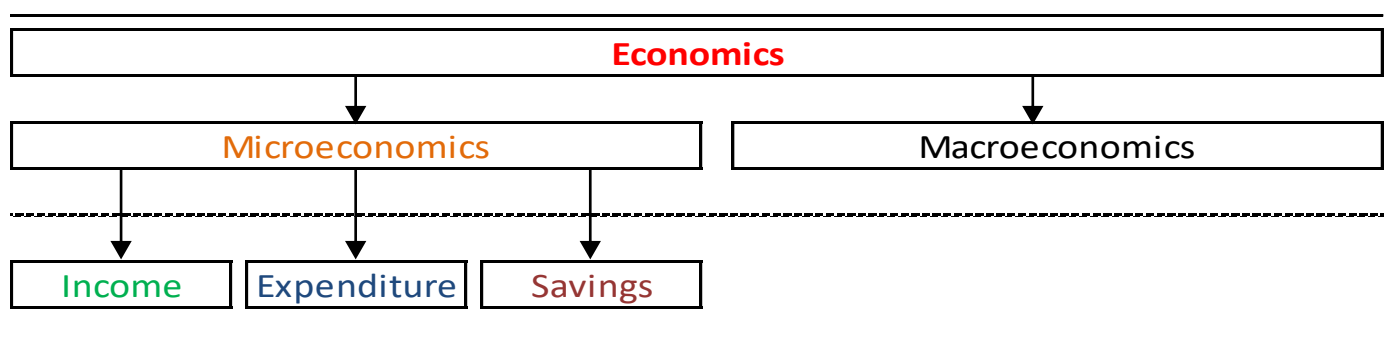

What is your monthly income per month?

How much do you spend on rent or mortgage repayments per month?

How much do you spend on transport per month?

How much do you spend on health or medical insurance per month?

How much do you spend on school fees per month?

How much do you spend on subsistence and upkeep per month?

How much do you save per month?

Respondent's sex (if possible, please observe and record)

How old were you on your last birthday?

What is your level of education?

What is your occupation?

Figure 4: Showing the academic field-of-study (economics), its key components (microeconomics and macroeconomics), and its variables (income, expenditure, and savings) as well as questions for the data collection instrument

\section{Summary and conclusions}

The panels that assess research proposals and ethical considerations also review the accompanying data or information collection instruments. Among others, they are interested in why we have included certain questions in our instruments as well as why we think such questions will help us realise our research purpose. To answer these questions, we need to have a firm grip on how to explicitly identify, interrogate, link, and integrate qualitative attributes or quantitative variables that are key to the business and public administration research that we are pursuing. Therefore, in Section 5, this paper proposes five complimentary and overlapping tasks to explicitly identify, interrogate, and integrate attributes and variables that are key to the research that we are pursuing. First, we should use our research questions as well as where applicable the accompanying research hypotheses or research propositions to identify the qualitative attributes or quantitative variables, if not both, that are key to the research that we are pursuing. Second, we should adopt and adapt attributes and variables from research that is similar to the research that we are pursuing. Third, we should then use appropriate academic literature to interrogate the attributes and variables that we have identified, adopted and adapted within the academic field-of-study that is appropriate to the research that we are pursuing. Therefore, before interrogating these attributes and variables, we should initially interrogate the appropriate academic field-of-study and its components to provide an academic discourse or context. Academic contextualisation may provide for us to theoretically and, in some cases, practically understand our research attributes and variables. Fourth, we should then use our theoretical and practical understanding of our research attributes and variables to decide the questions that we should include in our data and information collection instruments. Fifth, we should also use our understanding of our research attributes and variables to determine the most appropriate frameworks, theoretical or otherwise, that we should use to interpret our empirical research results.

Before making the proposals that we share in the preceding paragraph, in Section 2, we interrogated attributes and variables are in the context of research data or information collection instruments. We argue that there is 
little emphasis, if present, on theoretical interrogation of attributes and variables and yet such an interrogation is key to the research that we are pursuing is an important subcomponent of the conceptual framework. Further, the theoretical discussion of attributes or variables should be explicit linked to the various components and subcomponents of our research proposal or report. It is important to do so and we have justified this assertion by pointing out the four 'golden threads' in researching and research reporting (Section 4).

To set the foundation of our paper, we have reviewed the six main components of a research report as well as the four processes in research (Section 3). We then zero in the seven subcomponents of the second component (the conceptual framework) of a research. More specifically, we locate the subcomponent in which we should interrogate literature on attributes and variables that are key to the research that we are pursuing. Ideally, it is in the fifth subcomponent of the conceptual framework-where we should discuss the attributes or variables that are key to the research that we are pursuing.

More generally, this paper argues that we should go beyond mere description of attributes and variables in the context of research data or information collection instruments. Instead, we should explicitly identify, interrogate, and integrate attributes and variables that are key to the research we are pursuing. This implies theoretically and practically understanding the attributes and variables that are key to the research that we are pursuing. Such a discussion will contribute to a rich conceptual framework of our research. Further, we should deliberately link the components, subcomponents, and elements of our research-a point that we argue for in Section 4. Therefore, in this paper, we provide for actualising the third of the four 'golden threads'. In essence, we should determine our research attributes and variables through interrogating (i.) the research questions and where applicable the accompanying research hypotheses or research propositions and (ii.) the 'theoretical' knowledge gap or empirical literature. Further, we should discuss our selected attributes and variables in (iii.) the context of the academic field-of-study that is appropriate to the research that we are pursuing. In turn, we should use our theoretical and practical understanding of our research attributes or variables to determine (iv.) the questions that we package in our data or information collection instruments and ( $v$.) the frameworks, theoretical or otherwise, that we should use to interpret our empirical research results.

\section{Acknowledgements}

I am grateful to our research students that took part in a series of devising seminars where we discussed conceptualising conceptual frameworks; establishing interpretive frameworks; and now identifying, interrogating, and integrating attributes and variables that are key to the research that we are pursuing. I am, however, most grateful to the students that applied these proposed approaches for providing feedback on what worked and worked really well as well as what did not work. Further, I would like to thank colleagues, notably Prof Pundy Pillay, for the helpful comments and encouraging remarks. Lastly, as always, the reviewers including my super niece Chilala Chicha for helping me fine-tune this article and reconcile my argument as well as perfect my write-up.

\section{References}

Arnold, R.D., \& Wade, J.P., 2015. A definition of systems thinking: A systems approach. Procedia Computer Science, 44, 669678.

Badenhorst, C., 2007. Research writing: Breaking the barriers. Pretoria: Van Schaik Publishers.

Bryman, A., 2016. Social research methods. Oxford university press.

Connelly, L. M., 2015. Research questions and hypotheses. Medsurg Nursing, 24(6), 435-436.

Crossan, M. M., \& Apaydin, M., 2010. A multi-dimensional framework of organizational innovation: A systematic review of the literature. Journal of management studies, 47(6), 1154-1191.

Farrugia, P., Petrisor, B.A., Farrokhyar, F. and Bhandari, M., 2010. Research questions, hypotheses and objectives. Canadian journal of surgery, 53(4), p.278.

Gharajedaghi, J., 2006. Systems thinking: Managing Chaos and Complexity: A Platform for Designing Business Architecture. Elsevier Inc.

Imenda, S., 2014. Is there a conceptual difference between theoretical and conceptual frameworks?.Journal of Social Sciences, 38(2), 185-195.

Kaur, S. P., 2013. Variables in research. Indian Journal of Research and Reports in Medical Sciences, 3(4), 36-38.

Kumar, R., 2018. Research methodology: A step-by-step guide for beginners. Sage.

Letsiri, C., \& Wotela, K., 2015. International movements, post-apartheid dispensations and illegal immigration into South Africa. TD: The Journal for Transdisciplinary Research in Southern Africa, 11(4), 99-117.

Li, J.J. and Ma, N., 2013. Discussing variables nature in internet of things growth. In Applied Mechanics and Materials (Vol. 409, pp. 1604-1607). Trans Tech Publications Ltd. 
Marzo-Navarro, M., Pedraja-Iglesias, M. and Vinzón, L., 2017. Key variables for developing integrated rural tourism. Tourism Geographies, 19(4), pp.575-594.

Neuman, L.W., 2013. Social research methods: Qualitative and quantitative approaches. Pearson Education.

Pushpanjali, K., Piddennavar, R. and Mohan, M., 2011. Art and science of questionnaire development. Public Health, 2011(18).

Ravitch, S. M., \& Riggan, M., 2012. Reason and Rigor: How Conceptual Frameworks Guide Research. Sage Publications.

Rocco, T.S., \& Plakhotnik, M.S., 2009. Literature reviews, conceptual frameworks, and theoretical frameworks: Terms, functions, and distinctions. Human Resources Development Review. 8(1), pp. 120-130.

Wotela, K., 2016. Towards a systematic approach to reviewing literature for interpreting public and business management research results. The Electronic Journal of Business Research Methods. 14(2), pp. 83-97.

Wotela, K., 2017a. Conceptualising Conceptual Frameworks in Public and Business Management Research, Paper presented at $16^{\text {th }}$ European Conference on Research Methodology for Business \& Management Studies, $22-23$ June 2017. Dublin, Ireland. Academic Conferences \& Publishing International Limited.

Wotela, K., 2017b. Towards an outcomes-based approach to a 'research strategy, design, procedure and methods' chapter for business and public administration research. Journal of Public Administration. 52(Special Issue 1), pp. 223-246.

Wotela, K., 2018. Presenting empirical research results and discussing research findings in business and public administration, Paper presented at $17^{\text {th }}$ European Conference on Research Methodology for Business \& Management Studies, 12-13 July 2018. Rome, Italy on. Academic Conferences \& Publishing International Limited.

Wotela, K., 2019. Towards conceptualising business and public administration research augmented by analysing the physical research context, the research problem, and the research knowledge gap, Paper presented at $4^{\text {th }}$ International Conference on Public Administration and Development Alternatives (IPADA), $3^{\text {rd }}-5^{\text {th }}$ July 2019. Ekurhuleni, South Africa. International Conference on Public Administration and Development Alternatives.

Zhao, W., Wu, R. and Liu, H., 2016. Paper recommendation based on the knowledge gap between a researcher's background knowledge and research target. Information processing \& management, 52(5), pp.976-988. 